\title{
The intensification of gas-liquid flows with a periodic, constricted oscillatory-meso tube
}

\author{
N. Reis ${ }^{a}, *$ P.C. Mena ${ }^{b}$, A.A. Vicente ${ }^{a}$, J.A. Teixeira ${ }^{a}$, F.A. Rocha ${ }^{b}$ \\ ${ }^{a}$ IBB_-Institute for Biotechnology and Bioengineering, Centro de Engenharia Biológica, Universidade do Minho, Campus de Gualtar, 4710-057 Braga, Portugal \\ ${ }^{\mathrm{b}}$ ISR-P_Instituto de Sistemas e Robótica do Porto, Faculdade de Engenharia da Universidade do Porto, Rua Dr. Roberto Frias, $4200-465$ Porto, Portugal
}

Received 21 April 2007; received in revised form 24 August 2007; accepted 10 September 2007

Available online 20 September 2007

\begin{abstract}
The experimental study of gas dispersion in a vertical periodically, constricted, oscillatory meso-tube (OMT) is herein presented. Water was continuously pumped through the OMT in the laminar flow regime along with an oscillatory flow component superimposed into the net flow in a range of fluid oscillation frequency $(f)$ and centre-to-peak amplitude $\left(x_{0}\right)$ of $0-20 \mathrm{~s}^{-1}$ and $0-3 \mathrm{~mm}$, respectively, in the presence of a very low superficial gas velocity $\left(0.37 \mathrm{~mm} \mathrm{~min}^{-1}\right)$. Bubble images were recorded with a CCD camera and analysed with Visilog ${ }^{\circledR}$ software. A bimodal distribution of bubble size was in general observed but the bubble size was found strongly dependent on the oscillatory flow mixing conditions imposed into the fluid. A number fraction of micro-bubbles (with an equivalent diameter, $D_{\text {eq }}$, equal or bellow $0.2 \mathrm{~mm}$ ) up to $60 \%$ was generated with increasing values of $x_{0}$ (i.e. $3 \mathrm{~mm}$ ) and values of $f$ in the range $10-15 \mathrm{~s}^{-1}$. Furthermore, it is demonstrated that the Sauter mean diameter, $D_{32}$, and the specific interfacial area, $a$, can be fined tune by setting both $f$ and $x_{0}$ in this studied range. The high number fraction of micro-bubbles was concluded to have a positive impact in enhancing the liquid-side mass transfer coefficient, $k_{L}$. Globally, the differences in bubbles sizes were found to play a marginal effect in the global enhancement of the $k_{L} a$ in the meso-tube in comparison with the intensive contact experimented by the bubbles rising in the oscillatory flow. The higher order of magnitude of the $k_{L}$ values found in this work (up to $0.0021 \mathrm{~m} \mathrm{~s}^{-1}$ ) is promising for running numerous industrial gas-liquid flows processes through smaller and better, while aeration of biotransformations can be run more efficiently, as supported by our recent proof-of-concept studies carried out in the platform.
\end{abstract}

(C) 2007 Elsevier Ltd. All rights reserved.

Keywords: Constricted tube; Oscillatory flow; Aeration; Bubble; Mass transfer; Laminar flow

\section{Introduction}

In the last few years chemical and biochemical engineers have started acknowledging the advantages of carrying out reactions at small scale. Although plant design is traditionally based in scaled-out equipment, recent developments in scale-down platforms are powering the process intensification of industrial processes because of the important key advantages presented by these technologies.

The intensification of gas-liquid processes is particularly challenging because one must assure an efficient contacting for promoting the mass transfer between a gaseous and a liquid phases with very distinct densities. Limitations of mass and

\footnotetext{
* Corresponding author.

E-mail address: nunoreis@deb.uminho.pt (N. Reis).
}

heat transfer as well as hydrodynamics (fluid dynamics) should be substantially reduced in comparison with the intrinsic kinetics of the phenomena involved. In particular, the enhancement of the volumetric mass transfer coefficient $\left(k_{L} a\right)$ in gas-liquid systems requires the ability to engineer enhanced mass transfer coefficients, $k_{L}$, and maximum specific interfacial area, $a$. This involves the controlling of dependencies between a large set of hydrodynamic and physical parameters, including agitation intensity, sparger configuration, bubble size, bubble velocity and residence time, gas hold-up and superficial gas-velocity, etc. This is highly demanding especially for scale-down systems because of reduction in process residence times.

Process intensification (PI) describes the strategy of making dramatic reductions in plant volume in order to meet a certain production objective. PI offers the possibility to develop and carry out chemical, pharmaceutical and biochemical reactions 
in a sustainable way and with higher selectivity. The size of the equipment is adapted to the reaction, following the motivation of "doing better with less" (Ramshaw, 1999; Stankiewicz and Moulijn, 2000). Accordingly, microprocess engineering is changing the classical philosophy in plant engineering by developing "green technologies", where the number of side products is reduced and the selectivity and efficiency increased. Consequently, plant volume is shrunk and hazardous inventory reduced. Plant capital and operational costs (especially those related with cleaning) are also significantly reduced. In fact, "smaller" is a sign of "better" as demonstrated by several works and reviewed in detail (Hessel and Lowe, 2003a-c). PI is intimately related with the miniaturisation and the generation of enhanced force fields and is presently the major driving force for the development of "meso-technologies" (Pieters et al., 2006, 2007).

Gas-liquid flow is widely used in chemical and biochemical engineering, e.g. in gas-liquid catalytic reactions, fermentations and photosynthesis by micro-organisms. Extensive research has been carried out for decades in platforms conventionally used in large-scale, such as air-lift reactors, bubbles columns and stirred tanks. As most of gas-liquid processes are mass-transfer limited, a high demand still exists for innovation in continuous gas-liquid reactor designs as seen from the number of patents assigned (e.g., Schutte and Eickhoff, 2002).

Investigations of micro-technologies for gas-liquid contacting have been mainly performed in technically accepted reactor concepts, namely micro-packed bed reactor, falling-film microreactor and micro bubble column (Hessel and Lowe, 2003b). The high specific interfaces created in these micro-technologies are the key design for their improved performance in gas-liquid reactions. In particular, the micro-bubble column (Haverkamp et al., 2006) resembles the flow regimes in conventional bubble columns (a major established gas-liquid contacting technology) only for very low gas and liquid velocities. However, the small residence times (in the order of few seconds, as often the micro-channels are limited to few centimetre) require very high specific gas-liquid interfaces in order to speed up reactions. The bubbly mode is difficult to reproduce experimentally due to dosing problems at very small flow rates, thus increases of $a$ are obtained through operation at high gas and high liquid velocities. Consequently, it becomes difficult to set up the flow patterns maximising the specific gas-liquid interface (i.e., annular flow).

The oscillatory motion of a fluid through a set of periodic constrictions has demonstrated effective contacting and improvement of mass transfer rates in multiphase systems since Van Dick's (1935) work. However different designs were developed based on such technology. The oscillatory flow reactor (OFR) (Mackley, 1991) has proved to be the most popular design, consisting basically in a tube provided with periodic constrictions (with variable geometric configurations) where fluid pulsations (oscillations) are induced by a piston(s) installed in the tube inlet or at both ends moving sinusoidally along time. The strong radial mixing generated by the vortex rings (detaching from the narrow constrictions during flow reversal) enhances the contact between immiscible phases and improves the residence time distribution in the tube essentially for laminar net flows (Mackley and $\mathrm{Ni}, 1991$ ). Thus, the OFR naturally presents strategic advantages in PI (e.g., Harvey et al., 2003). Additionally, studies in small internal-diameter tubes (few millimetres diameter) have shown dispersed droplets and bubbles flowing through narrow, periodic-constrictions in a particular way, namely, with reduced mean rising velocities and considerable deformations when passing through the constrictions (Graham and Higdon, 2000; Hemmat and Borhan, 1996; Muradoglu and Gokaltun, 2004; Tsai and Miksis, 1994). This contributes to an enhancement of the mean residence time and specific interfacial area for dispersed phases.

This work characterises a recently presented (Reis et al., 2005) meso-technology as a gas-liquid contactor for the intensification of mass transfer rates from a dispersed gas at a very low gas flow velocity. A small diameter tube provided with narrow, periodic constrictions and operated in oscillatory flow conditions (here mentioned as the oscillatory meso-tube; OMT) was operated with a continuous liquid flow rate and a gas phase (air) sparged at a constant, small flow rate. The bubble size and the bubble size distribution (BSD) were determined using image analysis and here in presented for different combinations of fluid oscillation frequency and centre-to-peak amplitude. A significant impact of the oscillatory mixing intensity on the fine control of BSD, specific bubble area, residence time of bubbles (i.e. gas hold-up) and liquid-side mass transfer coefficient is demonstrated, highlighting potential applications of the OMT in processes with low, efficient requirements of a dissolved gas, as found in the aeration of cell cultures.

\section{Materials and methods}

\subsection{Experimental setup}

The configuration of the OMT was previously presented (Reis et al., 2005) and is illustrated in Fig. 1. It consists in a $4.4 \mathrm{~mm}$ internal diameter $(d)$ tube provided with narrow, smooth-periodic constrictions. The average inter-constrictions spacing $(L)$ is $\sim 13 \mathrm{~mm}$ and the mean constrictions diameter $\left(d_{c}\right)$ is $\sim 1.6 \mathrm{~mm}$.

Gas-liquid flow experiments were run as follows. A 350-mm length OMT (with and approximate internal volume of $4.5 \mathrm{ml}$ ) was positioned vertically and connected to a hard tubing in the bottom through a $T$-piece, allowing the continuous pumping of a constant flow rate $\left(Q_{L}=1.58 \mathrm{ml} \mathrm{min}^{-1}\right)$ of distilled water through the bottom of OMT with a peristaltic pump. This corresponds to a mean superficial liquid velocity, $U_{L}=2.1 \mathrm{~mm} \mathrm{~s}^{-1}$, based on the mean cross section tube diameter of $4.0 \mathrm{~mm}$. The distilled water was passed through a reservoir thus (eliminating the fluid pulsations from the peristaltic pump) and a valveless, rotating piston pump (CKCRH0, Fluid Metering Inc., New York) working in a closed-loop. The ceramic piston was driven by a stepper motor and the charge-discharge motion through a single revolution reproduced very well a sinusoidal oscillation in the fluid. The fluid oscillation frequency $(f)$ was controlled by setting the speed in the motor driver while the centre-to-peak amplitude $\left(x_{0}\right)$ was controlled by setting the 
volume-per-stroke of the ceramic piston. This configuration allowed a precise control of $f$ and $x_{0}$ in the range of $0-20 \mathrm{~s}^{-1}$ and 0-3 mm, respectively.

A $1 \mathrm{~mm}$ external diameter syringe needle was inserted within the first cavity in the bottom of the OMT through the $T$ connector and conveniently sealed. A continuous gas flow rate of compressed air $\left(Q_{G}\right)$ was passed through the epidemical needle and adjusted to $0.28 \mathrm{ml} \mathrm{min}^{-1}$ with a needle valve and afterwards confirmed with an electronic low gas flow meter; this corresponded to a mean superficial gas velocity $\left(U_{G}\right)$ of $\sim 0.37 \mathrm{~mm} \mathrm{~s}^{-1}$. No other gas distribution method was tried as the mass transfer under oscillatory flow mixing is dominated by the fluid flow patterns and is independent of the gas distributor, as concluded by Oliveira and $\mathrm{Ni}$ (2004). As the gas void fraction was very small (because of the small ratio $Q_{G} / Q_{L}$ ) the velocity field of the liquid phase was observed to not be significantly affected by the presence of a gas phase. Furthermore, the small bubbles were observed to follow the flow patterns of the liquid phase.

A Perspex-optical box filled with glycerol was then fitted and sealed in the mid-height of the OMT and the background illuminated with a halogen lamp, as illustrated in Fig. 1. A CCD camera with a faster shutter speed (up to $10000 \mathrm{~s}^{-1}$ ) was positioned in the front of the optical box and images with size $768 \times 576$ pixels continuously acquired and saved to a PC in TIF format at a frequency of $1 \mathrm{~s}^{-1}$. Although possible, a higher frequency of acquisition presented the disadvantage of overweighting bubbles rising with a small velocity (i.e. bubbles with a mean diameter higher than $d_{c}$ ) thus appearing in consecutive images. The time typically necessary for to complete the image acquisition procedure was $\sim 50 \mathrm{~min}$. The image acquisition system allowed a resolution of 39 pixels per millimetre, being appropriat for the analysis of bubbles sizes of $0.1 \mathrm{~mm}$ and above.

Prior to starting the experiments (i.e. before opening the gas-inlet valve), an image was acquired and saved as the "background". Bubble images were then taken at 20 different combinations of $x_{0}$ and $f$, namely $0.5,1.0,2.0$ and $3.0 \mathrm{~mm}$ and $3,7.5,10,15$ and $20 \mathrm{~s}^{-1}$, respectively (Table 1).For each combination of $f$ and $x_{0}$ about 3000 images were taken and resulted in the worse case in 595 bubbles being analysed. This was concluded to be sufficient for the BSD be independent in relation to the number of bubbles analysed (Fig. 2).

\subsection{Bubble size distribution and mean bubble size}

Image analysis of bubbles was carried out with a Visilog 5.4, a Graphical User Interface based image processing packaging available from Noesis, Velizy, France. Image analysis procedure started with the individual subtraction of the "background" to each TIF image, resulting in a binary image. Then, a series of thresholds, filters and dilations/erosions were applied and the most important bubbles' geometric parameters determined with Visilog. The most important parameter quantifying the size of each individual bubble is the equivalent spherical bubble diameter, $D_{\text {eq }}$, which is determined from the projected $\left(A_{\text {proj }}\right)$

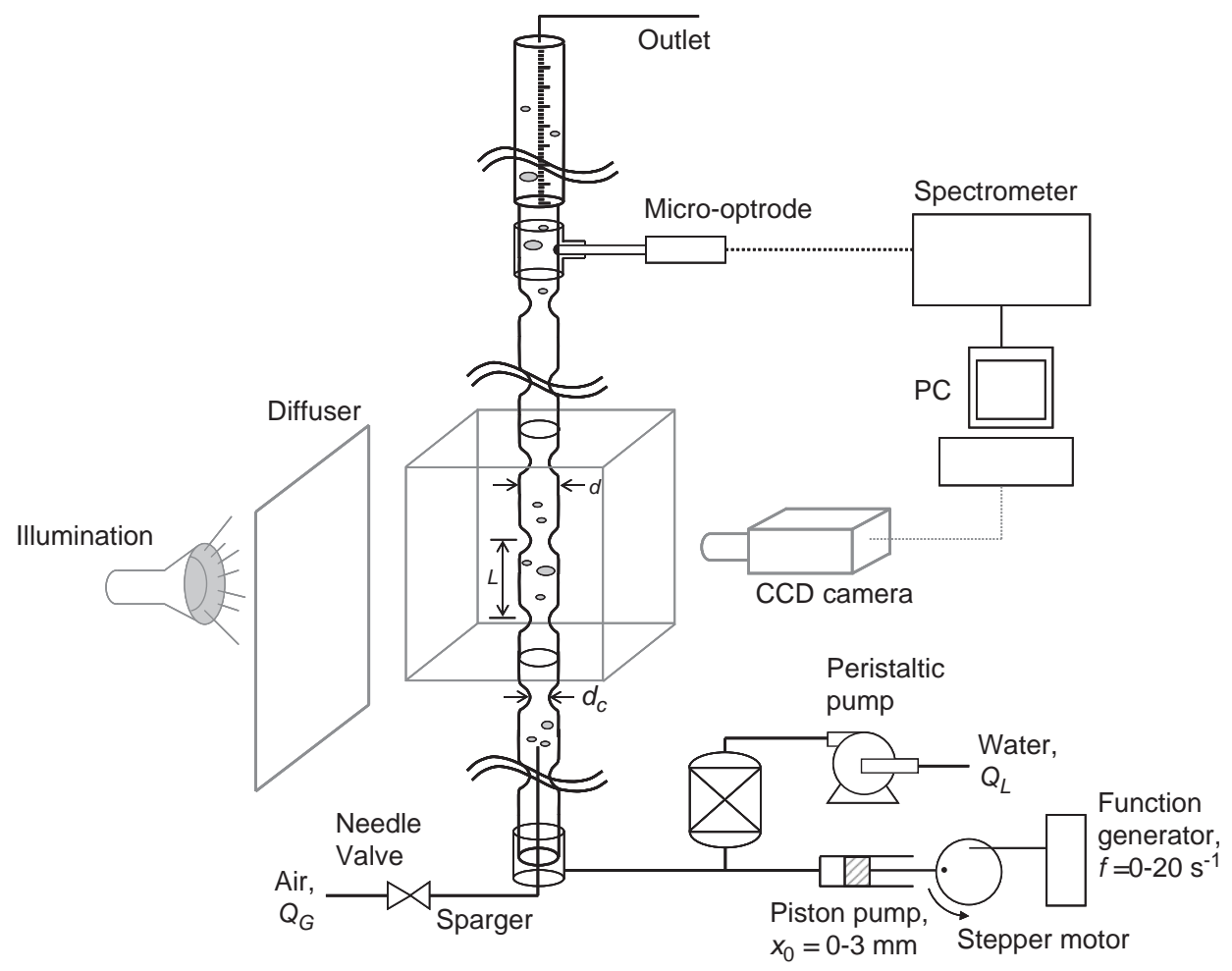

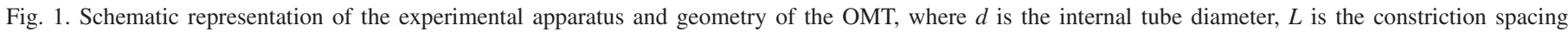
and $d_{c}$ is the free tube diameter in the constriction. 
Table 1

Summary of fluid oscillations conditions used in this work: frequency, $f$, centre-to-peak amplitude, $x_{0}$, respective maximum liquid oscillatory flow velocity, $u_{\max }=2 \pi f x_{0}$; summary of mean values for gas hold-up $\left(\varepsilon_{G}\right)$ in the OMT as calculated from Eq. 3. Values of volumetric mass transfer coefficient $\left(k_{L} a\right)$ determined for gas-liquid flow in a vertical OMT at $U_{L}=2.1 \mathrm{~mm} \mathrm{~s}^{-1}$ and $U_{G}=0.37 \mathrm{~mm} \mathrm{~s}^{-1}$

\begin{tabular}{lcrll}
\hline$x_{0}(\mathrm{~mm})$ & $f\left(\mathrm{~s}^{-1}\right)$ & $u_{\max }\left(\mathrm{mm} \mathrm{s}^{-1}\right)$ & $k_{L} a\left(\mathrm{~s}^{-1}\right)$ & $\varepsilon_{G}( \pm 0.003)(-)$ \\
\hline 0 & 0 & 0 & 0.009 & 0.034 \\
0.5 & 3 & 9 & 0.008 & 0.026 \\
0.5 & 7.5 & 24 & 0.009 & 0.048 \\
0.5 & 10 & 31 & 0.001 & 0.035 \\
0.5 & 15 & 47 & 0.010 & 0.013 \\
0.5 & 20 & 63 & 0.010 & 0.039 \\
1 & 3 & 19 & 0.012 & 0.029 \\
1 & 7.5 & 47 & 0.012 & 0.026 \\
1 & 10 & 63 & 0.019 & 0.016 \\
1 & 15 & 94 & 0.030 & 0.032 \\
1 & 20 & 126 & 0.061 & 0.045 \\
2 & 3 & 38 & 0.014 & 0.030 \\
2 & 7.5 & 94 & 0.027 & 0.013 \\
2 & 10 & 126 & 0.046 & 0.013 \\
2 & 15 & 188 & 0.065 & 0.042 \\
2 & 20 & 251 & 0.088 & 0.051 \\
3 & 3 & 57 & 0.016 & 0.022 \\
3 & 7.5 & 141 & 0.039 & 0.013 \\
3 & 10 & 188 & 0.079 & 0.013 \\
3 & 15 & 283 & 0.156 & 0.035 \\
3 & 20 & 377 & 0.155 & 0.042 \\
& & & &
\end{tabular}

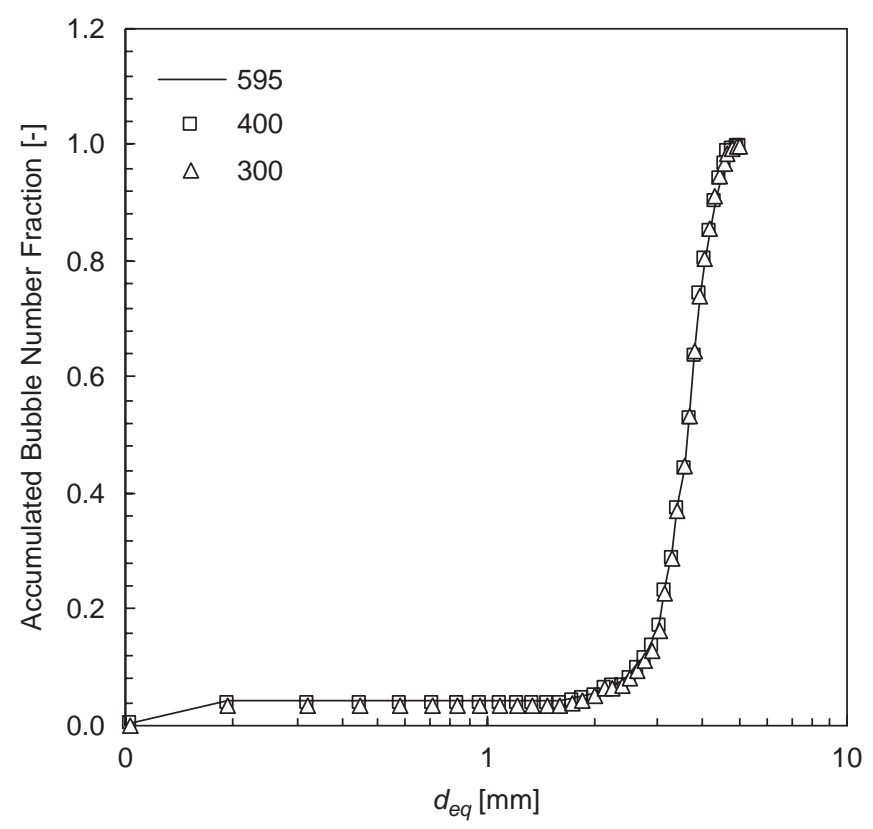

Fig. 2. Cumulative bubble size number for increasing number of bubbles analysed, namely 300, 400 and 595 (full experiment). Experiment carried out at $x_{0}=0.5 \mathrm{~mm}$ and $f=3 \mathrm{~s}^{-1}$.

according to

$D_{\mathrm{eq}}=\sqrt{\frac{4 A_{\mathrm{proj}}}{\pi}}$.
After analysing the full number of bubbles recorded for a certain combination of $f$ and $x_{0}$ a histogram was plotted in an excel's worksheet, with the number of bubbles distributed for increasing bubbles sizes at constant intervals (about $0.1 \mathrm{~mm}$ ) accordingly their value of $D_{\text {eq }}$. With this image analysis setup and post-processing procedure the minimum bubble size captured was $0.1 \mathrm{~mm}$. Finally, the bubble size fraction was plotted against the mean (interval) bubble size. This assured that several hundreds of bubbles were considered for the most frequent bubble sizes.

For processes involving mass transfer through an interfacial area, the BSD is well represented by the Sauter mean diameter $\left(D_{32}\right)$ which is given by

$D_{32}=\frac{\sum_{i} n_{i} \cdot D_{\mathrm{eq}_{i}}^{3}}{\sum_{i} n_{i} \cdot D_{\mathrm{eq}_{i}}^{2}}$.

\subsection{Gas hold-up}

The volume fraction of the gas phase (gas hold-up, $\varepsilon_{G}$ ) was measured in further experiments carried out at the same combinations of $f$ and $x_{0}$, by recording the changes in the liquid height in a fine scaled $( \pm 0.5 \mathrm{~mm})$ plain pipe $(6 \mathrm{~mm}$ i.d.) fitted to the top of the OMT, as represented in Fig. 1. It was continuously operated for 5-10 minutes at the desired combination of $f, x_{0}, Q_{L}$ and $Q_{G}$. Then, simultaneously the fluid level was fixed and recorded $\left(h_{1}\right)$ and both the fluid and the gas streams immediately stopped. After rinsing off all bubbles, the final liquid level was measured $\left(h_{2}\right)$ and $\varepsilon_{G}$ calculated from the volume variation by

$\varepsilon_{G}=\frac{h_{1}-h_{2}}{h_{1}}$.

This procedure was of easy implementation and allowed a high precision of $\varepsilon_{G}$ estimation in most experiments (a final error $\leqslant 5 \%$ ). A $6-\mathrm{mm}$ diameter was selected for the plain pipe to avoid bubble retention after the outlet of the OMT, thus maximizing the accuracy of $\varepsilon_{G}$ measurement.

\subsection{Specific interfacial area}

The specific gas-liquid contact area $(a)$ can be easily determined from the gas hold-up and the BSD as follows:

$a=6 \frac{\varepsilon_{G}}{D_{32}}$.

Values of $a\left(\mathrm{~m}^{2} \mathrm{~m}^{-3}\right)$ were used afterwards for estimating the liquid-side mass transfer coefficient $\left(k_{L}\right)$, based on volumetric oxygen mass transfer coefficients (product $k_{L} a$ ) determined at the same flow conditions.

\subsection{Estimating $k_{L}$ a values in the $O M T$}

The dissolved $\mathrm{O}_{2}$ (DO) saturation level in a volume of distilled water was set to zero by sparging it with nitrogen for $30 \mathrm{~min}$. The peristaltic pump was then turned on, the gas valve 
was opened and the fluid oscillations started. The DO saturation level was monitored at the outlet of the OMT with a micro-optrode (attached to a flow cell and previously calibrated in $0 \%$ and $100 \%$ DO saturation levels) until a stable readout was achieved (representing the DO saturation level for steady case). Typical time to achieve this final "steady-state" was about 10-15 hydraulic residence times, i.e. 30-45 min. The micro-optrode was a special fibre optic fluorescence microprobe ( $1.5 \mathrm{~mm}$ diameter $\mathrm{O}_{2}$ micro-optrode) provided with SMA connectors (Avs-oxyprobe-1.5, Avantes, Eerbeek, The Netherlands), dip-coated with a ruthenium complex immobilised in a sol-gel matrix.

Values of $k_{L} a$ were then estimated from mass balances to both the liquid and the gas phases and using an appropriate DO transport model for the continuous, oscillatory gas-liquid flow in the OMT. The liquid phase was modelled with a 1-D dispersion flow model. This model was previously validated from residence time studies with the liquid phase and the axial dispersion coefficients for every combination of $f$ and $x_{0}$ previously estimated (Reis et al., 2004). Estimated $k_{L} a$ values are summarised in Table 1. Further details concerning the procedure for estimating $k_{L} a$ are given elsewhere (Reis, 2006).

\section{Results and discussion}

The oscillatory movement superimposed on the liquid flow effectively has proved to be an effective strategy for breaking the bubbles in the OMT. At steady flow conditions (i.e. in absence of fluid oscillations) or in the presence of smooth fluid oscillations (i.e. $x_{0} \leqslant 0.5 \mathrm{~mm}$ and $f \leqslant 3 \mathrm{~s}^{-1}$ ) the gas sparging resulted in the formation of bubbles with a mean diameter similar to the mean internal diameter $d$ of the OMT (i.e. $4.4 \mathrm{~mm}$ ). More insightful oscillations are effective on decreasing the bubble size, as shown in Fig. 3. This effect is stronger for values of $f$ in the range of $10-15 \mathrm{~s}^{-1}$. The oscillatory movement also resulted in the enhancement of bubbles contacting due to an increase in mixing power, but coalescence was not observed and presumably did not occur because of the high velocity of bubbles imposed by the reversing movement (the peak fluid oscillation velocities-summarised in Table 1 -are actually 1 to 2 orders of magnitude higher than the mean superficial liquid velocity). This is illustrated in Fig. 3c and further illustrated in Fig. 4. The bubble's shape and the bubble's dynamics were both significantly affected by the oscillatory flow. Bubbles interacted very intensively with the walls, in particular with the narrow constrictions, slowing their rising motion. Due to the reversing movement, bubbles are also kept longer in the OMT and increase their mean residence time (Clift et al., 1978).

The significant bubble deformation observed at the higher values of $x_{0}$ (as previously shown in Fig. $3 \mathrm{c}$ and d) contributed to an enhancement in bubble surface area. This is considered as having a beneficial effect upon the gas-liquid mass transfer. Bubbles with a $D_{\text {eq }}<d_{c}$ (i.e. $4.4 \mathrm{~mm}$ ) were spheroids with small deformations (with a deformation ratio below 1.3, when referenced to Prolate's shape), but bubbles with a $D_{\text {eq }}>d_{c}$ presented a much-pronounced deformation.

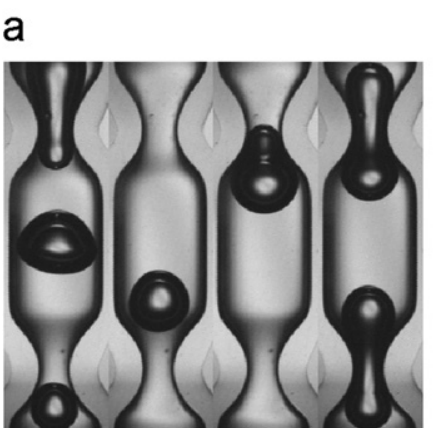

b

C

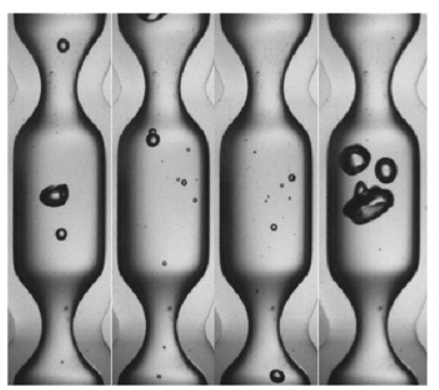

d
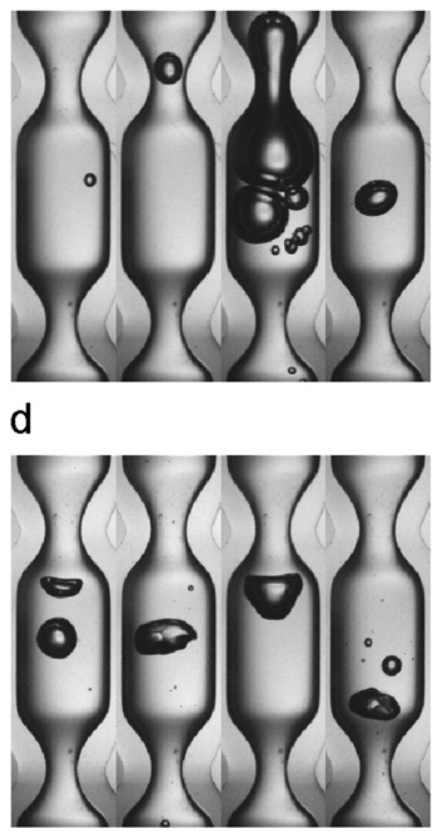

$5 \mathrm{~mm}$

Fig. 3. Typical shape of bubbles rising through the smooth, narrow constrictions in a vertical OMT, operated at $U_{L}=2.1 \mathrm{~mm} \mathrm{~s}^{-1}$ and $U_{G}=0.37 \mathrm{~mm} \mathrm{~min}^{-1}$ and different fluid oscillation conditions: (a) $0.5 \mathrm{~mm}$, $3 \mathrm{~s}^{-1}$; (b) $3 \mathrm{~mm}, 3 \mathrm{~s}^{-1}$; (c) $3 \mathrm{~mm}, 10 \mathrm{~s}^{-1}$; (d) $3 \mathrm{~mm}, 15 \mathrm{~s}^{-1}$.

Fig. 5 summarises the Sauter mean diameter of the bubbles for different values of $f$ and $x_{0}$. The operation at the upper values of $x_{0}$ and intermediate values of $f$ (in the range of $7.5-15 \mathrm{~s}^{-1}$ ) facilitates breaking of the bubbles in to small and more uniform sizes, although a fast reciprocating movement $\left(f=20 \mathrm{~s}^{-1}\right)$ resulted in the formation of bubbles with larger sizes. The effect of oscillatory flow upon the control of bubble sizes in the OMT is clearer in terms of BSD curves. Fig. 6a shows the plots of BSDs (represented as a bubble number fraction) for constant, increasing values of $x_{0}$ and a constant $f=15 \mathrm{~s}^{-1}$, while Fig. $6 \mathrm{~b}$ represents the plots of BSDs at increasing values of $f$ and a constant $x_{0}=3.0 \mathrm{~mm}$. A bimodal distribution of bubble sizes was in general observed. The bubble number fraction of the first mode (corresponding to micro-bubbles with an average $D_{\text {eq }} \approx 0.2 \mathrm{~mm}$ ) is remarkable and presents a maximum value at $x_{0}=2-3 \mathrm{~mm}$ and $f=10-15 \mathrm{~s}^{-1}$. The second mode relative to bubble sizes about one order of magnitude higher (i.e. $D_{\text {eq }} \approx 1.5-3.5 \mathrm{~mm}$ ) was eliminated by the fluid oscillations in that range, producing a rather uniform, unimodal bubble size distribution. This is thought to be due the higher shear strain rates generated in the OMT, breaking the large bubbles into several tiny bubbles. In the limit case herein studied the bubble number fraction has reached a maximum value around 0.60 , at $f=15 \mathrm{~s}^{-1}$ and $x_{0}=3 \mathrm{~mm}$.

The large number fraction of micro-bubbles was concluded having a strong impact on the interfacial area in the OMT. The values of $a$ represented in Fig. 7 were calculated using the gas hold-up (summarised in the table and in the range of 0.01-0.05) 
a

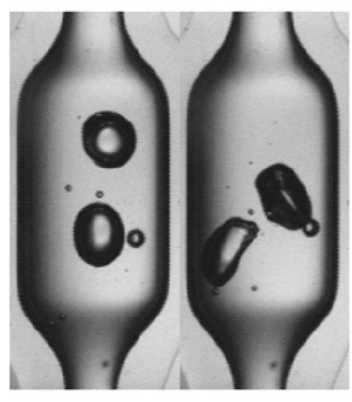

I b

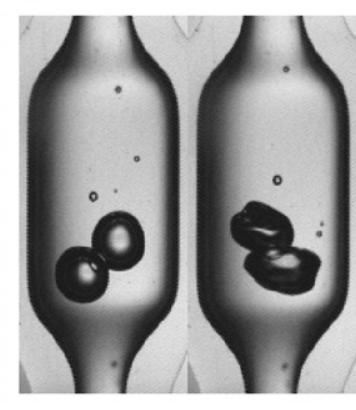

II
C

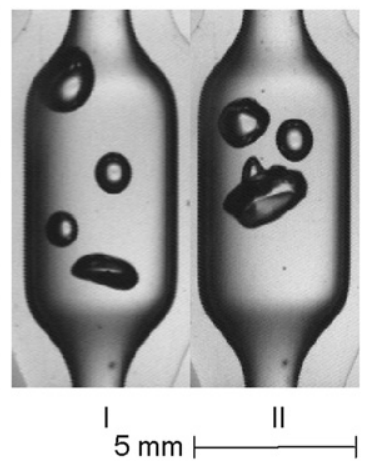

Fig. 4. Bubbles motion within the vertical OMT at intensive oscillatory flow conditions $\left(f=10 \mathrm{~s}^{-1}, x_{0}=3 \mathrm{~mm}\right)$. Bubbles images were taken at two consecutive shots (I and II) $1 / 3000 \mathrm{~s}$ apart.

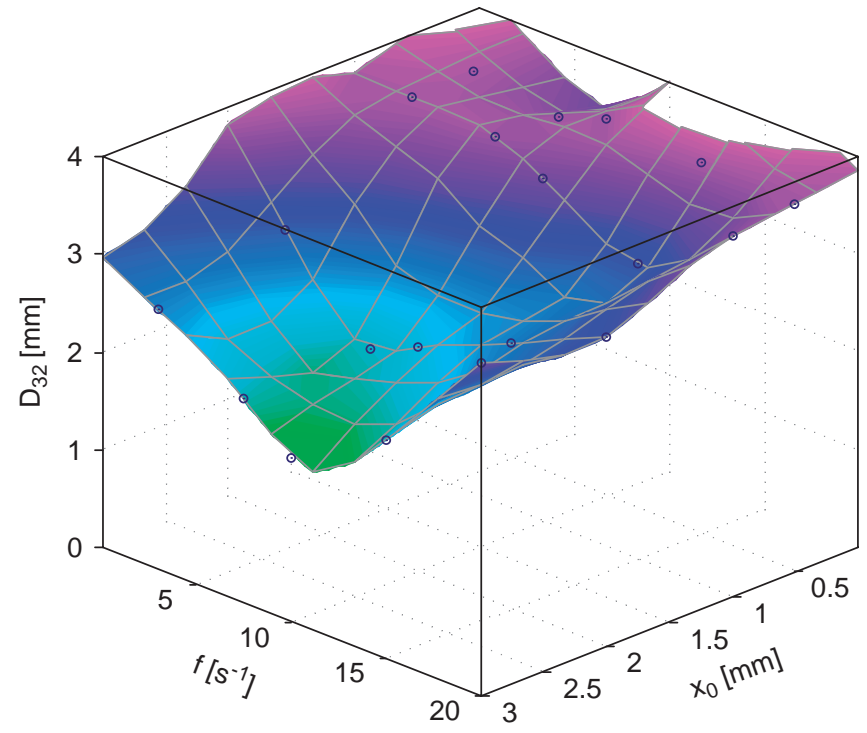

Fig. 5. Sauter mean diameter $\left(D_{32}\right)$ in the vertical OMT as a function of fluid oscillation frequency $(f)$ and centre-to-peak amplitude $\left(x_{0}\right)$, at $U_{L}=2.1 \mathrm{~mm} \mathrm{~s}^{-1}$ and $U_{G}=0.37 \mathrm{~mm} \mathrm{~min}^{-1}$.

and the Sauter mean diameter, accordingly Eq. (4). Although values of $a$ have a slight experimental error (a maximum error of $\pm 10 \%$ was estimated, following the determination of gas hold-ups and $D_{32}$ ) the high degree of scattering (as $a$ varies in the range of $20 \pm 2.0$ to $97 \pm 19.4 \mathrm{~m}^{2} \mathrm{~m}^{-3}$ ) had a significant contribution from the large number of micro-bubbles assuring a decrease in $D_{32}$.

The effect of $f$ and $x_{0}$ on $k_{L}$, is summarised in Fig. 8. The values of $k_{L}$ increase with both $f$ and $x_{0}$, exhibiting a significant degree of scattering because of the accumulated experimental errors, namely from $D_{32}, \varepsilon_{G}$ and most especially $k_{L} a$. The $k_{L} a$ values summarised in Table 1 were estimated based on triplicate measurements of dissolved $\mathrm{O}_{2}$ saturation levels in the OMT. The standard deviation of $k_{L} a$ values very significant decrease of the 5-20\%, and a sensibility analysis allowed concluding $k_{L}$ values in Fig. 8 having an error up to $\pm 30 \%$.
The increase in $k_{L}$ as seen in Fig. 8 is due to an increase in fluid mixing power according to models currently available for predicting the power input in oscillatory flow reactors (e.g. the quasi-steady approach of Jealous and Johnson, 1955). The maximum value of $k_{L}=0.0021 \pm 0.00056 \mathrm{~s}^{-1}$ was obtained for $f=20 \mathrm{~s}^{-1}$ and $x_{0}=3 \mathrm{~mm}$. This value is roughly two orders of magnitude higher than those typically reported for bubble columns and represents more than a 2-fold increasing in comparison with $k_{L}$ values obtained in a conventional OFR. Oliveira and $\mathrm{Ni}(2004)$ reported a maximum $k_{L} \approx 0.00079 \mathrm{~s}^{-1}$ for a $50 \mathrm{~mm}$ i.d. OFR, using a maximum $f=10 \mathrm{~s}^{-1}, x_{0}=10 \mathrm{~mm}$ and $U_{G}=4.2 \mathrm{~mm} \mathrm{~s}^{-1}$. This $k_{L}$ enhancement is explained by an increase in fluid mixing and power input in the OMT in comparison with the OFR. The smaller baffle free diameter $\left(d_{c} / d=0.36\right.$ and 0.48 for the OMT and OFR, respectively) and the higher number of constrictions (76 and 13 for the OMT and OFR, respectively) contributes to an $\sim 15$-fold increasing in the specific power input for the same value of $f$ and $x_{0}$, accordingly the quasi-steady approach of Jealous and Johnson (1955). Also in comparison with the bubble column, the higher renewal rate of the liquid at the gas-liquid interface results in very significant decrease of the liquid-side film thickness. This result is not surprising at all because one knows the secondary flow (helical or oscillatory) in free-rising bubbles leads substantially to an enhancement in gas-liquid mass transfer rates (Clift et al., 1978).

In large scale gas-liquid contactors the $k_{L}$ values are substantially lower for small bubbles than for large ones because of differences in fluid recirculation at the surface, with a transition regime in between, which is characterised by a steep increase in $k_{L}$ (Robinson and Wilke, 1974). An increase in mixing intensity improves the mass transfer rates in gas-liquid flows and systematically results in to the generation of many tiny bubbles with a high specific interfacial area. However, the positive effect of $D_{32}$ on $a$ is counterbalanced with the negative effect of $D_{32}$ in $k_{L}$, thus the enhancements in $k_{L} a$ are usually insignificant at constant gas flow rates. This is the case of the conventional OFR (Oliveira and Ni, 2004). Neverthless, Fig. 9a shows an opposite scenario of $D_{32}$ versus $k_{L}$ for the OMT. A smaller value of $D_{32}$ actually means a higher number of dispersed, tiny bubbles at a constant gas flow rate, with a higher interaction 
a

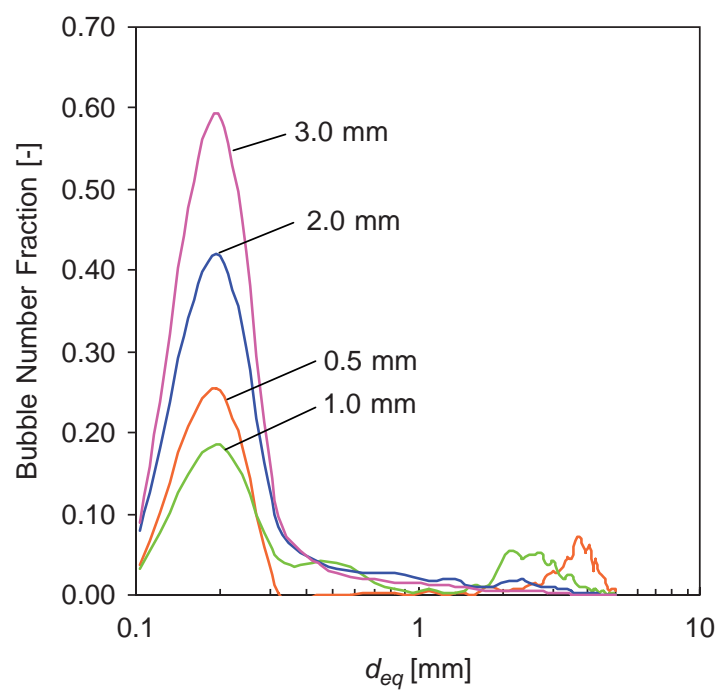

b

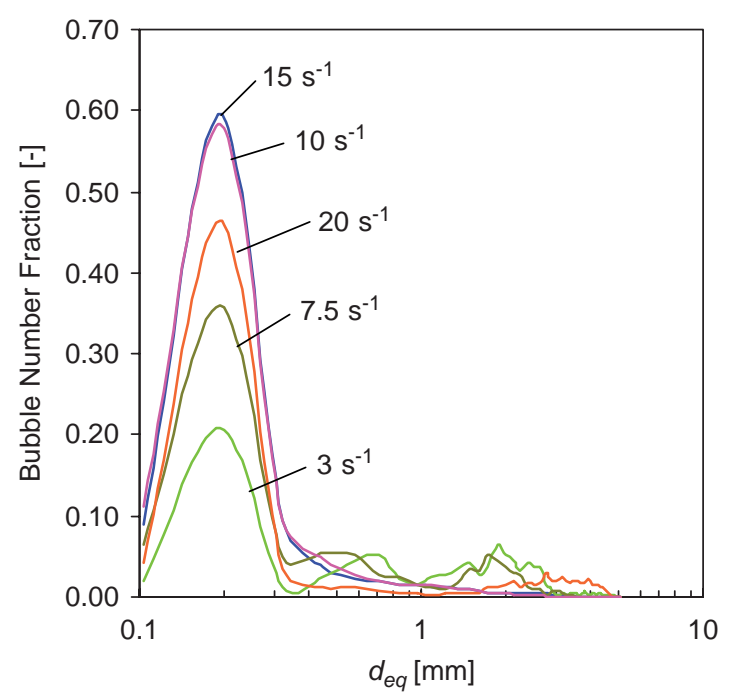

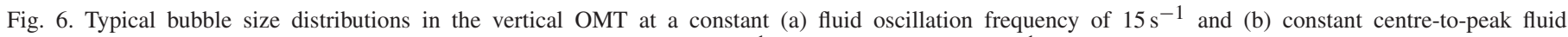
oscillation amplitude of $3 \mathrm{~mm}$, when operated at constants $U_{L}=2.1 \mathrm{~mm} \mathrm{~s}^{-1}$ and $U_{G}=0.37 \mathrm{~mm} \mathrm{~min}^{-1}$.

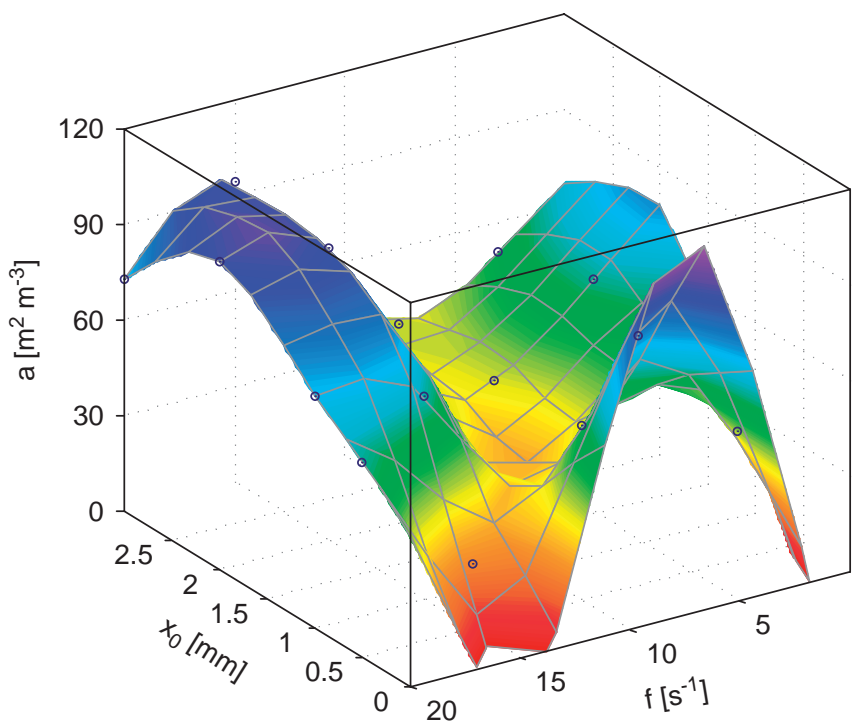

Fig. 7. Specific interfacial area $\left(\mathrm{m}^{2} \mathrm{~m}^{-3}\right)$ in the vertical OMT as a function of both fluid oscillation frequency $(f)$ and centre-to-peak amplitude $\left(x_{0}\right)$, for continuous gas-liquid flow at $U_{L}=2.1 \mathrm{~mm} \mathrm{~s}^{-1}$ and $U_{G}=0.37 \mathrm{~mm} \mathrm{~min}^{-1}$.

between the bubbles and the walls, thus resulting in a decrease in the liquid-side film thickness. Futhermore, high values of $f$ present a higher renewal rate of the liquid at the gas-liquid interface, thus positively improving the $k_{L}$. Actually, a faster reciprocating movement (with a higher $f$ ) enhances the number of collisions of the dispersed phases upon the enhancement of mass transfer rates. In every upward and downward movement of the fluid inside the OTM the high velocity of the fluid crossing the smooth, periodic constriction results in the flow separation and the generation of vortex rings. These 3-D structures are fluid elements with higher energy and momentum, representing volumes fluid with high velocities and vorticities

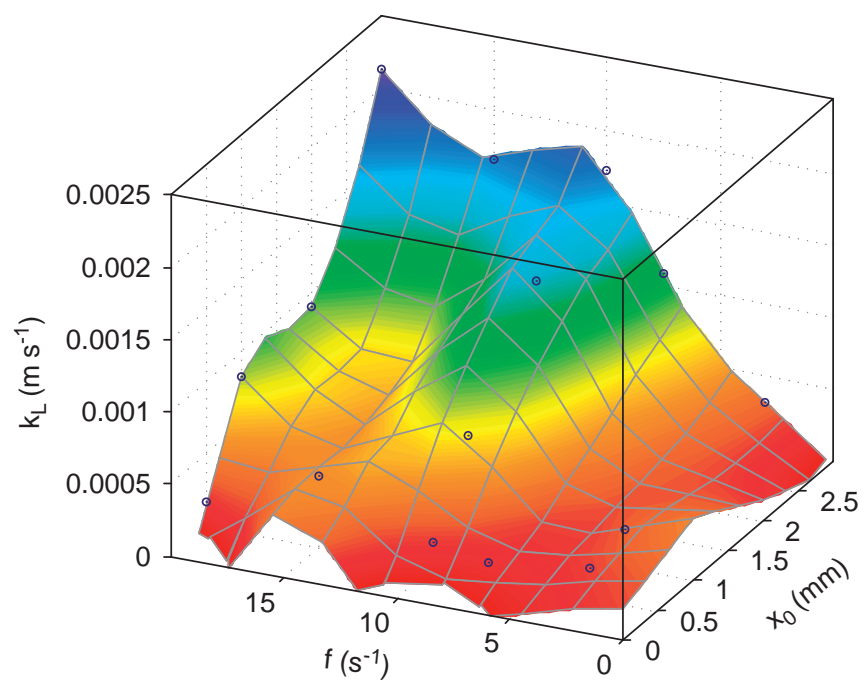

Fig. 8. Effect of both the fluid oscillation frequency $(f)$ and centre-to-peak amplitude $\left(x_{0}\right)$ on the liquid side mass transfer coefficient $\left(k_{L}\right)$ for continuous gas-liquid flow at $U_{L}=2.1 \mathrm{~mm} \mathrm{~s}^{-1}$ and $U_{G}=0.37 \mathrm{~mm} \mathrm{~min}^{-1}$ in the vertical meso-tube.

propagating along the fluid bulk towards the walls, where they usually dissipate. During this dynamic movement the vortex rings carry out the dispersed phase and endorse strong interactions with the liquid phase.

Globally, the bubble size appears playing a marginal role in the control of mass transfer within the OMT when compared with the effect of oscillatory fluid mixing or gas hold-up, as the higher values of $k_{L}$ and $k_{L} a$ were obtained at the most intensive oscillatory flow conditions (i.e. $f=20 \mathrm{~s}^{-1}$ and $x_{0}=3 \mathrm{~mm}$ ), with a corresponding high value of $D_{32} \approx 4 \mathrm{~mm}$. Fig. $9 \mathrm{~b}$ shows the contribution of decreasing $D_{32}$ on the enhancement of $a$ is not linear (as expected from Eq. (4), accordingly $a$ depends 

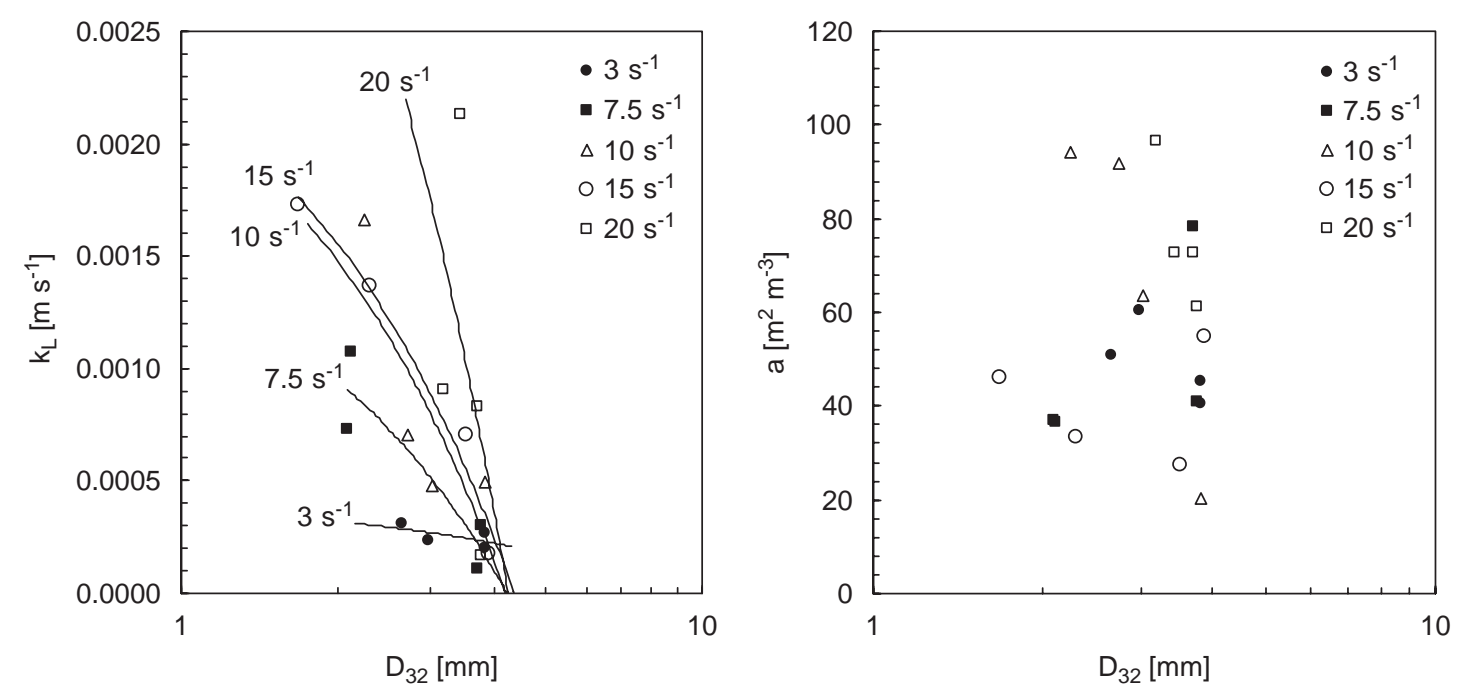

Fig. 9. Effect of Sauter mean diameter $\left(D_{32}\right)$ on (a) liquid side mass transfer $\left(k_{L}\right)$ and (b) specific interfacial area $(a)$ at constant fluid oscillation frequencies in the range of $3-20 \mathrm{~s}^{-1}$, in the continuous vertical OMT.

also of $\varepsilon_{G}$ ) and not necessarily positive. The bubble breakage results in tiny bubbles (thus to a decrease in $D_{32}$ ) that could theoretically freely rise along the narrow constrictions. However, the oscillatory movement is very important and effective on increasing the mean residence time of these small bubbles, by keeping them inside the OMT and assuring values of $\varepsilon_{G}$ very significant.

In short, the results for $k_{L}$ and $a$ herein presented demonstrate the oscillatory movement superimposed on a liquid flow has a major impact in the intensification of gas-liquid mass transfer phenomena occurring in a periodically, constricted OMT at low superficial gas velocities.

\section{Conclusions}

Gas-liquid flow was herein studied for a periodically constricted meso-tube operating at oscillatory flow conditions. This has demonstrated superior performance for gas-liquid contacting and for general intensification of mass transfer rates from a dispersed gas at a very low gas superficial velocity.

The bubble size and the bubble size distribution were determined through image analysis at different combinations of and $x_{0}$ superimposed into a continuous, laminar net flow. A fine control of BSD, specific bubble area, gas hold-up, and liquidside mass transfer coefficient was obtained in the presence of fluid oscillations, and supports the application of this EOMT in processes having a small but efficient composition demand of a dissolved gas, as it was the case in proof-of-concept aerobic biotransformations previously carried out in this platform (Reis et al., 2006).

A bimodal distribution of bubble size was in general observed. The number fraction of micro-bubbles (with an average size of $1.5-3.5 \mathrm{~mm}$ approximately) increased with both $f$ and $x_{0}$. The second mode relative to larger bubbles with an average size of $1.5-3.5 \mathrm{~mm}$ practically disappears for the higher values of frequency (say $f \geqslant 10 \mathrm{~s}^{-1}$ ) and centre-to-peak amplitudes $\left(x_{0} \geqslant 2.0 \mathrm{~mm}\right)$ herein tested. For the limit cases studied the number fraction of micro-bubbles reached a value around $60 \%$.

The large amount of micro-bubbles was concluded having a strong impact on the specific interfacial area in the OMT and the results suggested that this influence increases with both $f$ and $x_{0}$.

Values of $k_{L}$ were improved at the most intensive fluid mixing conditions, and were two orders of magnitude higher than those typically reported for bubble columns and represents more than a 2-fold increasing in relation to the $k_{L}$ values reported for a $50 \mathrm{~mm}$ internal diameter tube provided with sharp edges (the conventional OFR's geometry).

This work demonstrates that the intensive reciprocating mixing experimented by bubbles rising in a periodically, constricted-meso tube is the major factor controlling the global enhancement of $k_{L} a$ in this scale-down platform.

\section{Notation}

\begin{tabular}{|c|c|}
\hline$a$ & specific interfacial area, $\mathrm{m}^{2} \mathrm{~m}^{-3}$ \\
\hline$A_{\text {proj }}$ & projected bubble area, $\mathrm{mm}^{2}$ \\
\hline$d$ & internal tube diameter, $\mathrm{mm}$ \\
\hline$d_{c}$ & free diameter in the constriction, $\mathrm{mm}$ \\
\hline$D_{\text {eq }}$ & equivalent bubble diameter, $\mathrm{mm}$ \\
\hline$D_{32}$ & Sauter mean diameter, $\mathrm{mm}$ \\
\hline$f$ & fluid oscillation frequency, $\mathrm{s}^{-1}$ \\
\hline$k_{L}$ & liquid-side mass transfer coefficient, $\mathrm{m} \mathrm{s}^{-1}$ \\
\hline$k_{L} a$ & volumetric mass transfer coefficient, $\mathrm{s}^{-1}$ \\
\hline$L$ & constrictions spacing, $\mathrm{mm}$ \\
\hline$Q_{G}$ & gas flow rate, $\mathrm{ml} \mathrm{min}^{-1}$ \\
\hline$Q_{L}$ & liquid flow rate, $\mathrm{ml} \mathrm{min}^{-1}$ \\
\hline
\end{tabular}


$U_{G} \quad$ mean superficial gas velocity, $\mathrm{mm} \mathrm{s}^{-1}$

$U_{L} \quad$ mean superficial liquid velocity, $\mathrm{mm} \mathrm{s}^{-1}$

$x_{0} \quad$ centre-to-peak fluid oscillation amplitude, $\mathrm{mm}$

\section{Greek letters}

$\varepsilon_{G} \quad$ gas hold-up

\section{Acknowledgement}

N. Reis acknowledges the financial support of Fundação para a Ciência e Tecnologia (FCT), Portugal.

\section{References}

Clift, R., Grance, J.R., Weber, M.E., 1978. Bubbles, Drops, and Particles. Academic Press, New York.

Graham, D.R., Higdon, J.J.L., 2000. Oscillatory flow of droplets in capillary tubes. Part 2. Constricted tubes. Journal of Fluid Mechanics 425, 55-77.

Harvey, A.P., Mackley, M.R., Seliger, T., 2003. Process intensification of biodiesel production using a continuous oscillatory flow reactor. Journal of Chemical Technology and Biotechnology 78 (2-3), 338-341.

Haverkamp, V., et al., 2006. Hydrodynamics and mixer-induced bubbles formaion in micro bubble columns with single and multiple-channels. Chemical Engineering \& Technology 29 (6), 1015-1026.

Hemmat, M., Borhan, A., 1996. Buoyancy-driven motion of drops and bubbles in a periodically constricted capillary. Chemical Engineering Communications 148 (50), 363-384.

Hessel, V., Lowe, H., 2003a. Microchemical engineering: components, plant concepts, user acceptance-part i. Chemical Engineering and Technology (26), 13-24.

Hessel, V., Lowe, H., 2003b. Microchemical engineering: components, plant concepts, user acceptance-part ii. Chemical Engineering and Technology (26), 391-408.

Hessel, V., Lowe, H., 2003c. Microchemical engineering: components, plant concepts, user acceptance-part iii. Chemical Engineering and Technology (26), 531-544.

Jealous, A.C., Johnson, H.F., 1955. Power requirements for pulse generation in pulse columns. Industrial and Engineering Chemistry 47 (6), $1168-1169$.
Mackley, M.R., 1991. Process innovation using oscillatory flow within baffled tubes. Chemical Engineering Research \& Design 69 (3), 197-199.

Mackley, M.R., Ni, X., 1991. Mixing and dispersion in a baffled tube for steady laminar and pulsatile flow. Chemical Engineering Science 46 (12), 3139-3151.

Muradoglu, M., Gokaltun, S., 2004. Implicit multigrid computations of buoyant drops through sinusoidal constrictions. Journal of Applied Mechanics 71 (6), 857-865.

Oliveira, M.S.N., Ni, X.W., 2004. Effect of hydrodynamics on mass transfer in a gas-liquid oscillatory baffled column. Chemical Engineering Journal 99 (1), 59-68.

Pieters, B., et al., 2006. The impact of microtechnologies in chemical and pharmaceutical production processes. In: 2006 Spring National Meeting, Orlando, FL.

Pieters, B., Andrieux, G., Eloy, J.-C., 2007. The impact of microtechnologies on chemical and pharmaceutical production processes. Chemical Engineering \& Technology 30 (3), 407-409.

Ramshaw, C., 1999. Process intensification and green chemistry. Green Chemistry (1), G17-G19.

Reis, N., 2006. Novel oscillatory flow reactors for biotechnological applications. Ph.D. Thesis, University of Minho, Braga.

Reis, N., et al., 2004. Residence times and mixing of a novel continuous oscillatory flow screening reactor. Chemical Engineering Science 59 (22-23), 4967-4974.

Reis, N., et al., 2005. Fluid mechanics and design aspects of a novel oscillatory flow meso-reactor. Chemical Engineering Research \& Design 83 (A4), 357-371.

Reis, N., et al., 2006. Proof-of-concept of a novel micro-bioreactor for fast development of industrial bioprocesses. Biotechnology and Bioengineering 95 (4), 744-753.

Robinson, C.W., Wilke, C.R., 1974. Simultaneous measurement of interfacial area and mass transfer coefficients for a well-mixed gas dispersion in aqueous electrolyte solutions. A.I.Ch.E. Journal 20 (2), 285-294.

Schutte, R., Eickhoff, H., 2002. Process for carrying out gas-liquid reactions and continuous flow reactor for this purpose. United States Patent, USA, Degussa-Huls AG, Frankfurt (DE).

Stankiewicz, A.I., Moulijn, J.A., 2000. Process intensification: transforming chemical engineering. Chemical Engineering Progress 96 (1), 22-34.

Tsai, T.M., Miksis, M.J., 1994. Dynamics of a drop in a constricted capillary tube. Journal of Fluid Mechanics 274, 197-217.

Van Dick, W.J.D., 1935. Process and apparatus for intimately contacting fluids. United States Patent Office, USA. 